\title{
ULTRA-RELATIVISTIC EXPANSION OF IDEAL FLUID WITH LINEAR EQUATION OF STATE
}

\author{
V. I. Zhdanov, M. S. Borshch \\ Taras Shevchenko National University of Kyiv \\ (Received February 25, 2005; in final form June 15, 2005)
}

\begin{abstract}
We study solutions of the relativistic hydrodynamic equations, which describe spherical or cylindrical expansion of ideal fluid. We derived approximate solutions involving two arbitrary functions which describe the asymptotic behaviour of expanding fireballs in ultra-relativistic limit. In the case of a linear equation of state $p(\varepsilon)=\kappa \varepsilon-c_{1},(0<\kappa<1)$ we show that the solution may be represented in form of an asymptotic series in negative powers of radial variable; recurrence relations for the coefficients are obtained. This representation is effective if $\kappa>1 /(2 J+1)(J=2$ for spherical expansion and $J=1$ for the cylindrical one); in this case the approximate solutions have a wave-like behaviour.
\end{abstract}

Key words: relativistic hydrodynamics, gamma-ray bursts, relativistic fireballs.

PACS number(s): $47.75 .+\mathrm{f}$

\section{INTRODUCTION}

The problem of the relativistic ideal fluid expansion arises, e.g., in the theory of gamma-ray bursts, that represent the most powerful explosions in the Universe [1]. The other applications are related to hydrodynamical theory of multiparticle production [2]. Here the relativistic ideal fluid model is utilized to describe the behaviour of relativistic fireballs. A thorough study of this problem involves complicated 3-D numerical simulations. However, considerable information may be obtained from analytical studies, which deal typically with one-dimensional flows having spherical, cylindrical and plane symmetry $[2,3]$. One of such well-known results is due to Blandford \& McKee [4] who studied self-similar ultra-relativistic gas expansion following a strong shock. The Blandford $\&$ McKee solution is widely used for interpretation of the gamma-ray bursts and their afterglows [1]. Certain drawbacks of this solution have been pointed out in Ref. [5].

It should be noted that the investigation of ultrarelativistic asymptotics of ideal flows is rather specific; it requires some care to provide a valid approximation. For example, if we leave only the main terms in the hydrodynamical equations in ultra-relativistic limit, these become degenerate (see Sec. II). To be sure about the approximation involved, one must study the higher order corrections in the equations. Evidently, it is desirable to have a sufficiently general form of the solutions that contains the necessary number of arbitrary functions. This is important, e.g., in order to describe models of an expanding fluid with different radial density profiles.

In this connection we propose an approximation method to construct general solutions that describe ultra-relativistic radial expansion of an ideal fluid with the necessary degree of accuracy. The most detailed result is obtained in the case of a linear equation of state (EOS); in this case we represent the solution in the form of an asymptotic series (Sec. III), which is workable at least for a sufficiently stiff EOS. These results are compared in Sec. IV with self-similar solutions.

\section{MAIN EQUATIONS}

The equations of motion of an ideal relativistic fluid follow from the conservation laws (see, e.g., [2,3])

$$
\partial_{\nu} T^{\mu \nu}=0
$$

for the energy-momentum tensor $T^{\mu \nu}=(p+$ $\varepsilon) u^{\mu} u^{\nu}-p g^{\mu \nu} ; u^{\nu}$ is the fluid four-velocity, $g^{\mu \nu}=$ $\operatorname{diag}(1,-1,-1,-1), p$ is the pressure, $\varepsilon$ is the invariant energy density, and the light speed $c=1$. We confine ourselves to an EOS of the form $p=p(\varepsilon)$. Some kind of the continuity equation must be added in a more general case of the two-parametric EOS.

In case of cylindrical $(J=1)$ and spherical $(J=2)$ symmetry of the flow we deal with only two independent variables (time $t$ and radial variable $r$ ). The equations take on the form [2], [3]:

$$
\begin{aligned}
& \frac{\partial}{\partial t}\left[\left(u^{t}\right)^{2} \varepsilon+\left(u^{r}\right)^{2} p\right]+\frac{\partial}{\partial r}\left[u^{t} u^{r}(p+\varepsilon)\right] \\
& =-\frac{J(p+\varepsilon)}{r} u^{t} u^{r}, \\
& \frac{\partial}{\partial t}\left[u^{t} u^{r}(p+\varepsilon)\right]+\frac{\partial}{\partial r}\left[\left(u^{t}\right)^{2} p+\left(u^{r}\right)^{2} \varepsilon\right] \\
& =-\frac{J(p+\varepsilon)}{r}\left(u^{r}\right)^{2},
\end{aligned}
$$

where $u^{r}$ is the radial component of the four-velocity; the other spatial four-velocity components equal zero. 
In ultra-relativistic limit $u^{r} \approx u^{t} \gg 1$. But if we leave in Eqs. (1) and (2) only the higher order terms in $u^{r}$, then both from (1) and (2) we have the same equation

$$
\begin{aligned}
& \frac{\partial}{\partial t}\left[\left(u^{t}\right)^{2}(\varepsilon+p)\right]+\frac{\partial}{\partial r}\left[\left(u^{t}\right)^{2}(\varepsilon+p)\right] \\
& =-\frac{J}{r}\left[\left(u^{t}\right)^{2}(\varepsilon+p)\right]
\end{aligned}
$$

that is, we have a certain degeneracy. We can determine $\left(u^{t}\right)^{2}(\varepsilon+p)$ from this equation, but we cannot determine the velocity and the energy density separately. This shows that we cannot neglect the terms looking at first sight as less important.

\section{FIRST APPROXIMATION}

Further we use the substitution $u^{t}=u+1 /(4 u)$, $u^{r}=u-1 /(4 u)$. Then Eqs. (1) and (2) yield:

$$
\begin{aligned}
& \frac{\partial}{\partial t}\left[\left(u^{2}+\frac{1}{16 u^{2}}\right)(\varepsilon+p)+\frac{\varepsilon-p}{2}\right] \\
& +\frac{\partial}{\partial r}\left[\left(u^{2}-\frac{1}{16 u^{2}}\right)(\varepsilon+p)\right] \\
& =-\frac{J(p+\varepsilon)}{r}\left(u^{2}-\frac{1}{16 u^{2}}\right), \\
& \frac{\partial}{\partial t}\left[\left(u^{2}-\frac{1}{16 u^{2}}\right)(\varepsilon+p)\right] \\
& +\frac{\partial}{\partial r}\left[\left(u^{2}+\frac{1}{16 u^{2}}\right)(p+\varepsilon)-\frac{\varepsilon-p}{2}\right] \\
& =-\frac{J(p+\varepsilon)}{r}\left(u^{2}+\frac{1}{16 u^{2}}-\frac{1}{2}\right) .
\end{aligned}
$$

Consider the sum and difference of Eqs. (3) and (4) in new variables $\alpha=t-r, \beta=t+r$. We have

$$
\begin{aligned}
& \frac{\partial}{\partial \beta}(\varepsilon-p)+\frac{\partial}{\partial \alpha}\left(\frac{\varepsilon+p}{4 u^{2}}\right) \\
& =-\frac{J(\varepsilon+p)}{\beta-\alpha}\left(1-\frac{1}{4 u^{2}}\right) \\
& \frac{\partial}{\partial \beta}\left[u^{2}(\varepsilon+p)\right]+\frac{\partial}{\partial \alpha}\left(\frac{\varepsilon-p}{4}\right) \\
& =-\frac{J(\varepsilon+p)}{\beta-\alpha}\left(u^{2}-\frac{1}{4}\right)
\end{aligned}
$$

These are still exact equations.

In the ultra-relativistic approximation $u \gg 1$ we retain the terms $\sim u^{2}$ and zero orders in this value. Neglecting the terms containing $\sim 1 / u^{2}$, we have from (5)

$$
\frac{\partial}{\partial \beta}(\varepsilon-p) \approx-\frac{J(\varepsilon+p)}{\beta-\alpha}
$$

This equation can be solved, if we know the state equation $p=p(\varepsilon)$. In this case Eq. (7) yields

$$
(1-d p / d \varepsilon) \frac{\partial \varepsilon}{\partial \beta} \approx-\frac{J[\varepsilon+p(\varepsilon)]}{\beta-\alpha} .
$$

Let by definition

$$
\Phi(\varepsilon)=\exp \left[\int \frac{1-d p / d \varepsilon}{\varepsilon+p(\varepsilon)} d \varepsilon\right] .
$$

Then the solution of Eq. (8) may be represented as

$$
\varepsilon \approx \Phi^{-1}\left\{\frac{f(\alpha)}{(\beta-\alpha)^{J}}\right\}
$$

where $f(\alpha)$ is an arbitrary function. The validity of this approximate solution depends on the smallness of the terms in Eq. (5) that have been neglected, i. e., the approximation is valid if

$$
\left|\int d \beta(\beta-\alpha)^{J} \frac{\partial}{\partial \alpha}\left(\frac{\varepsilon+p}{4 u^{2}(\beta-\alpha)^{J}}\right)\right| \ll|f(\alpha)| .
$$

As we shall see in the next section this condition is satisfied, e.g., in case of a linear $\operatorname{EOS} p(\varepsilon)=\kappa \varepsilon-c_{1}$ if $1>\kappa>1 /(2 J+1)$ for $r \rightarrow \infty$ and bounded $\alpha$.

Eq. (6) can be written as

$$
\begin{aligned}
& (\beta-\alpha)^{-J} \frac{\partial}{\partial \beta}\left[u^{2}(\beta-\alpha)^{J}(\varepsilon+p)\right] \\
& =\frac{J(\varepsilon+p)}{4(\beta-\alpha)}-\frac{\partial}{\partial \alpha} \frac{\varepsilon-p}{4} .
\end{aligned}
$$

Using $\varepsilon$ from (9) we obtain $u$ from

$$
\begin{aligned}
& u^{2}(p+\varepsilon)(\beta-\alpha)^{J} \\
& =g(\alpha)+\int d \beta \frac{(\beta-\alpha)^{J}}{4}\left[\frac{J(\varepsilon+p)}{(\beta-\alpha)}-\frac{\partial}{\partial \alpha}(\varepsilon-p)\right],
\end{aligned}
$$

where $g(\alpha)$ is an arbitrary function. This enables us to find $\varepsilon$ and $u$ separately.

If the integral in the right-hand side of (10) converges for $\beta \rightarrow \infty$ and $\alpha$ remains bounded, then for sufficiently large $\beta \sim t$ we obtain that $u^{2}(\varepsilon+p)(\beta-\alpha)^{J}$ is a function only of $\alpha$. We shall see below that this is just the case of the linear EOS with $\kappa>1 /(2 J+1)$. In this case we have that the energy profile of the ultra-relativistic expanding 
shell $d E / d \alpha \approx(\varepsilon+p) u^{2} r^{J}$ remains almost constant for large values of $t$.

\section{HIGHER ORDERS OF ASYMPTOTIC SERIES FOR THE SOLUTIONS}

Consider the higher orders of approximation in case of a linear equation of state $p(\varepsilon)=\kappa \varepsilon-c_{1}$, where $\kappa=c_{0}^{2}<1, c_{0}$ is the speed of sound. We are looking for the asymptotic representation of the solution which is workable for bounded $\alpha=t-r$ and $t \rightarrow \infty$; this means also $\beta \sim r \rightarrow \infty$.

We put

$$
\begin{aligned}
& \varepsilon=\frac{\varphi(\alpha, \beta)}{(\beta-\alpha)^{a}}+c_{1} /(1+\kappa), \quad a=\frac{J(1+\kappa)}{1-\kappa}, \\
& u^{2}(\varepsilon+p)=\frac{\psi(\alpha, \beta)}{(\beta-\alpha)^{J}} .
\end{aligned}
$$

This substitution reduces set (5)-(6) to the form

$$
\begin{aligned}
& \frac{\partial \varphi}{\partial \beta}+\frac{(1+\kappa)^{2}}{4(1-\kappa)} \frac{1}{(\beta-\alpha)^{a-J}} \frac{\partial}{\partial \alpha}\left(\frac{\varphi^{2}}{\psi}\right) \\
& =-\frac{J \kappa(1+\kappa)^{2}}{(1-\kappa)^{2}} \frac{1}{(\beta-\alpha)^{a-J+1}} \frac{\varphi^{2}}{\psi}
\end{aligned}
$$

$$
\frac{\partial \psi}{\partial \beta}+\frac{1-\kappa}{4} \frac{1}{(\beta-\alpha)^{a-J}} \frac{\partial \varphi}{\partial \alpha}=0
$$

We shall look for a solution of the exact equations (11), (12) in the form of asymptotic series $(m, n=0,1,2, \ldots)$

$$
\begin{aligned}
& \varphi(\alpha, \beta)=\sum_{n, m} \frac{\varphi_{n, m}(\alpha)}{(\beta-\alpha)^{\gamma n+m}}, \\
& \psi(\alpha, \beta)=\sum_{n, m} \frac{\psi_{n, m}(\alpha)}{(\beta-\alpha)^{\gamma n+m}},
\end{aligned}
$$

where $\gamma=a-J-1$.

If we are looking for an asymptotic solution for $r \rightarrow \infty$, this representation is effective for $\gamma>0$, that is for $\kappa>1 /(2 J+1)$.

We also introduce

$$
\chi(\alpha, \beta)=\frac{\varphi^{2}}{\psi}=\sum_{n, m} \frac{\chi_{n, m}(\alpha)}{(\beta-\alpha)^{\gamma n+m}}
$$

the coefficients of these series may be expressed be means of $\varphi_{n, m}, \psi_{n, m}$. Moreover, $\chi_{n, m}$ depends only upon $\varphi_{n^{\prime}, m^{\prime}}$, $\psi_{n^{\prime}, m^{\prime}}$ with $n^{\prime} \leq n, m^{\prime} \leq m$.

We now substitute the above representations for $\varphi$, $\psi, \chi$ into Eqs. (11), (12) to find recurrence relations for $\varphi_{n, m}, \psi_{n, m}$.

For $n \geq 1, m \geq 1$ we have

$$
\begin{aligned}
(\gamma n+m) \varphi_{n, m} & =\frac{(1+\kappa)^{2}}{4(1-\kappa)}\left\{\frac{d \chi_{n-1, m}}{d \alpha}+\left[\gamma(n-1)+m-1+\frac{4 J \kappa}{(1-\kappa)}\right] \chi_{n-1, m-1}\right\} \\
(\gamma n+m) \psi_{n, m} & =\frac{1-\kappa}{4} \frac{d \varphi_{n-1, m}}{d \alpha}+\frac{1-\kappa}{4}[\gamma(n-1)+m-1] \varphi_{n-1, m-1} .
\end{aligned}
$$

For $n=0, m=1,2, \ldots$

$$
\varphi_{0, m}=\psi_{0 . m}=0,
$$

whence also $\chi_{0, m}=0$.

For $m=0, n=1,2, \ldots$

$$
n \gamma \varphi_{n, m}=\frac{(1+\kappa)^{2}}{4(1-\kappa)} \frac{d \chi_{n-1,0}}{d \alpha}
$$

and

$$
\gamma n \psi_{n, 0}=\frac{1-\kappa}{4} \frac{d \varphi_{n-1,0}}{d \alpha}
$$

Using (15), (16), (17), (18) we can express all the coefficients of the series (13), (14) by means of $\psi_{00}(\alpha)$ and $\varphi_{00}(\alpha)\left(\psi_{00} \neq 0\right)$
Here we write expressions for some lowest order coefficients

$$
\begin{aligned}
& \varphi_{1,0}=\frac{(1+\kappa)^{2}}{4 \gamma(1-\kappa)} \frac{d \chi_{0,0}}{d \alpha}, \quad \psi_{1,0}=\frac{1-\kappa}{4 \gamma} \frac{d \varphi_{0,0}}{d \alpha}, \\
& \varphi_{2,0}=\frac{(1+\kappa)^{2}}{8 \gamma(1-\kappa)} \frac{d \chi_{1,0}}{d \alpha}, \quad \chi_{1,0}=2 \frac{\varphi_{1,0} \varphi_{0,0}}{\psi_{0,0}}-\frac{\psi_{1,0} \varphi_{0,0}^{2}}{\psi_{0,0}^{2}},
\end{aligned}
$$$$
\psi_{2,0}=\frac{(1+\kappa)^{2}}{2 \gamma^{2}} \frac{d^{2} \chi_{0,0}}{d \alpha^{2}}, \quad \varphi_{1,1}=\frac{J \kappa(1+\kappa)^{2}}{(1-\kappa)^{2}(\gamma+1)} \chi_{0,0}
$$

$\psi_{1, m}=0, m=1,2, \ldots$ 


\section{SELF-SIMILAR SOLUTIONS}

The representation of solution in the previous section allows us to obtain the coefficients of the series (13),(14) up to any given order. Therefore, if $\kappa>1 /(2 J+1)$, we may calculate an asymptotic representation of the solution with any desired accuracy for $r \rightarrow \infty$. Nevertheless, this does not mean that series (13) and (14) are convergent. Therefore it would be useful to check the results of the previous section using self-similar solutions when we deal with the associated ordinary differential equations.

We choose the similarity variable as $\xi=r / t$. In case of the $\operatorname{EOS} p=\kappa \varepsilon-c_{1} /(1+\kappa)$ set (1)-(2) leads to ordinary differential equations

$\xi \frac{d v}{d \xi}=J \kappa\left(1-v^{2}\right) \frac{\mu \xi\left(1-v^{2}\right)+v(1-v \xi)}{\left[(v-\xi)^{2}-\kappa(1-v \xi)^{2}\right]}$,

$\xi \frac{d \sigma}{d \xi}=J(1+\kappa) \frac{\mu \kappa+[1+(1-\kappa) \mu] v \xi-(1+\mu) v^{2}}{\left[(v-\xi)^{2}-\kappa(1-v \xi)^{2}\right]}$,

where $v=u^{r} / u^{t}=\left(4 u^{2}-1\right) /\left(4 u^{2}+1\right), \sigma=\ln \rho$, $\varepsilon=r^{\nu} \rho+c_{1} /(1+\kappa), \mu=\nu /[J(1+\kappa)]$.

A qualitative investigation of self-similar relativistic fluid flows with spherical and cylindrical symmetry can be found in [3]. Here we confine ourselves to asymptotic solutions of Eqs. (20), (21) near the boundary of the expanding fluid, where $u \gg 1, v \rightarrow 1$. If the fluid expands to vacuum, its boundary moves with the light speed (corresponding to $\xi=1$ ). Using the results of a qualitative invesigation in [3] for the phase plane $\{v, \xi\}$ it is easy to classify possible asymptotic solutions of Eqs. (20), (21) near this boundary for $\xi \rightarrow 1$ and to obtain their asymptotics.

In case of $\kappa>1 /(2 J+1)$ we have

$$
\begin{aligned}
& u^{2} \approx A_{1}(1-\xi)^{p_{1}}, \quad p_{1}=-\frac{2 \kappa J}{1-\kappa}, \\
& \varepsilon \approx A_{2} r^{\nu}(1-\xi)^{p_{2}}, \quad p_{2}=\nu+\frac{J(1+\kappa)}{1-\kappa},
\end{aligned}
$$

$A_{1}, A_{2}$ being arbitrary constants.

This corresponds to the results of the previous section, where one should assign

$$
\varphi_{00}(\alpha) \sim \alpha^{p_{2}}, \quad \psi_{00}(\alpha) \sim \alpha^{\nu+J} .
$$

If $\kappa<1 /(2 J+1)$, this type of solution is absent.

On the other hand, there is also a solution with asymptotics

$$
u^{2}=A_{ \pm}(1-\xi)^{-1}, \quad A_{ \pm}=(1-\kappa)^{-1}(B \pm \sqrt{D})
$$

where $B=1+\kappa+J \kappa(1+2 \mu)$,

$$
D=B^{2}+(1-\kappa)[(1+2 J) \kappa-1] \text {. }
$$

For $\kappa>1 /(2 J+1)$ it is easy to see that $A_{-}<0$, so only $A_{+}>0$ survives. For $\kappa<1 /(2 J+1)$ both $A_{-}$and
$A_{+}$are positive. Depending upon $\nu$ and $\kappa$, Eq. (24) may represent either a special solution or an infinite family of solutions. Some values of the parameters may lead to $\varepsilon \rightarrow \infty$ for $\xi \rightarrow 1$ and they must be matched to other solutions through the shock wave; the other parameters correspond to solutions that can be matched to vacuum $(\varepsilon \rightarrow 0)$. These asymptotics are not well-described by the finite number of terms in representation (13), (14) of the previous section.

\section{DISCUSSION}

We studied non-stationary ultra-relativistic hydrodynamic flows having spherical or cylindrical symmetry in the case of the equation of state $p=p(\varepsilon)$. The results may be applicable also to the case of a more general EOS $p=p(\varepsilon, S)$ if the fluid motion is isentropic ( $S=$ const). The solutions derived in Sec. IV (as well as the first order approximations of Sec. III) contain two arbitrary functions, therefore they represent some kind of a general solution of the equations of the fluid motion. However, the consideration of section $\mathrm{V}$ shows that they do not describe solutions of all possible types equally well in the ultra-relativistic limit. Formally we may use some of the above results to the case of arbitrary $\kappa \in(0,1)$, $p(\varepsilon)=\kappa \varepsilon-c_{1}$, for any ultra-relativistic case, but in certain limited domain of spatial variables, where the approximations work.

The approximations work in the best way in the case of the "stiff" equation of state: $\kappa>1 / 5$ in the case of a spherical expansion (this involves the important case $\kappa=1 / 3$ ), and $\kappa>1 / 3$ in the case of a cylindrical one. In these cases the representations of Sec. III are most effective for $t-r \ll t \rightarrow \infty$. The corresponding solutions exhibit the wave-like behaviour irrespectively of the energy profile of the expanding shell. The energy of the spherical/cylindrical shell, which expands almost with the speed of light, is approximately conserved. This does not mean, however, that this shell will contain all the energy of the expanding fluid. It would be interesting to study what is the part of the total energy carried by this expanding wave-like shell and to relate the shell profile to the initial conditions; this probably cannot be done without a numerical investigation. On the other hand, in the case of small values of $\kappa$ we may expect that the energy of the fluid is spread over all the values of $r \sim t$. The values of the speed of sound squared $c_{0}^{2}=1 /(2 J+1)$ may be considered as some bifurcation points which separate different regimes of ultra-relativistic flow.

The method of Section III allows one to derive formally the solutions up to any order of approximation. However, we do not make here any statement about the convergence of the series bearing in mind that the calculation of high orders involves higher order derivatives. Nevertheless, our results show that, in spite of some degeneracy in the main order of hydrodynamical equations in ultra-relativistic limit, we do not meet any problems like small denominators in the higher orders and in this sense we may be sure about the asymptotic properties of the solutions. 
[1] P. Meszaros, Ann. Rev. Astron. Astrophys. 40,137 (2002).

[2] Yu. P. Ochelkov et al., Reliativistskaia kinetika i gidrodinamika (Relativistic kinetics and hydrodynamics) (Atomizdat, Moscow, 1979) [in Russian].

[3] N. R. Sibgatullin, Kolebaniia $i$ volny $v$ sil'nykh gravitatsionnykh $i$ élektromagnitnykh poliakh (Oscillations and waves in strong gravitational and electromagnetic fields) (Nauka, Moscow, 1984) [in Russian].

[4] R. D. Blandford, C. F. McKee, Phys. Fluids 19, 1130 (1976).

[5] A. Gruzinov, preprint astro-ph/0012364 (2000).

\title{
УЛЬТРАРЕЛЯТИВІСТСЬКЕ РОЗШИРЕННЯ ІДЕАЛЬНОЇ РІДИНИ З ЛІНІЙНИМ РІВНЯННЯМ СТАНУ
}

\author{
В. I. Жданов, М. С. Борщ \\ Київсъкий начіоналъний університет імені Тараса Шевченка
}

Розглянуто розв'язки рівнянь релятивістської гідродинаміки, які описують сферичне або циліндричне розширення ідеальної рідини. Отримано наближені розв'язки з двома довільними функціями, які описують асимптотичну поведінку фаєрбола, що розширюється, в ульрарелятивістській границі. Для лінійного рівняння стану $p(\varepsilon)=\kappa \varepsilon-c_{1}(0<\kappa<1)$ показано, що розв'язок можна подати як асимптотичниий ряд за від'ємними степенями радіяльної змінної. Отримано рекурентні співвідношення для коефіцієнтів ряду. Це представлення ефективне за умови $\kappa>1 /(2 J+1)(J=2$ для сферичного та $J=1$ для циліндричного розширення); у цьому разі наближені розв'язки мають хвилеподібну поведінку. 